\title{
Publisher Correction: Combination of Chemical and Neurotrophin Stimulation Modulates Neurotransmitter Receptor Expression and Activity in Transdifferentiating Human Adipose Stromal Cells
}

Arthur A. Nery ${ }^{1} \cdot$ Ricardo L. Pereira ${ }^{1} \cdot$ Vinicius Bassaneze ${ }^{2} \cdot$ Isis C. Nascimento ${ }^{1} \cdot$ Lauren S. Sherman $^{3}$. Pranela Rameshwar ${ }^{3} \cdot$ Claudiana Lameu $^{1} \cdot$ Henning Ulrich ${ }^{1}$ (I)

(C) Springer Science+Business Media, LLC, part of Springer Nature 2019

Publisher Correction: Stem Cell Reviews and Reports https://doi.org/10.1007/s12015-019-09915-1

The original version of this article unfortunately contained mistakes. Several errors were introduced within Table 1. The correct version and presentation of the Table is as follows.

The Publisher regrets these mistakes.

The original article has been corrected.

The online version of the original article can be found at https://doi.org/ 10.1007/s12015-019-09915-1

Henning Ulrich

henning@iq.usp.br

1 Department of Biochemistry, Institute of Chemistry, University of São Paulo, São Paulo, Brazil

2 Heart Institute (InCor), University of São Paulo Medical School, São Paulo, Brazil

3 Division of Hematology/Oncology, Department of Medicine, New Jersey Medical School, Rutgers Biomedical and Health Sciences, Newark, NJ, USA 


\begin{tabular}{|c|c|c|c|c|c|}
\hline Receptors & $\begin{array}{l}\text { Activation } \\
\text { Mechanism }\end{array}$ & Agonist & Gene Target & Primer Forward Sequence & Primer Reverse Sequence \\
\hline & Ionotropic & Glutamate & GRIA2 & GCAGCGGGGGAGGTGATTCC & GCCACCAGCATTGCCAAACC \\
\hline \multirow[t]{6}{*}{ Glutamatergic } & & & GRIA3 & CAAAGGAGAGTGCGGCAGCG & CCATCATGGCCAGCCCCAGA \\
\hline & Metabotropic & & GRM2 & TGACGCGATGCGGCCAGTTA & TGTCAGCTGGGCGAAAGGGG \\
\hline & Ionotropic & Acetylcholine & CHRNA3 & ATCATGGAGACCAACCTGTG & AACTCTGCCCCACCATAGTC \\
\hline & & & CHRNAG & TGCTGACCAGCAAGGGGCAG & AGTTGCACAGCCCACACAGC \\
\hline & & & CHRNB2 & CTGCTCCTTGGCTTCGGCCT & AGATGCTCCACCAGCCGCTC \\
\hline & & & CHRNB4 & TTCCTGGTCGCCCTTTGCGG & GTGGCTGGGCGGATCAGGTT \\
\hline \multirow[t]{5}{*}{ Cholinergic } & Metabotropic & & CHRM1 & GGCACGCTGGCTTGTGA & TTCATGACGGAGGCATTGC \\
\hline & & & CHRM2 & TCGAACACACACGCACGAAT & CCCTGGCGCCTTTAGTACAG \\
\hline & & & CHRM3 & CTGTCCTATGCCGGGATCAT & TGTAACAAGGATGCAAAACA \\
\hline & & & CHRM4 & GCAGCTCGGGCAATCAGT & TCTCATAGCGATTGTGGGAT \\
\hline & & & CHRM5 & CTGGCCCAGCGGGAAAGGAA & TGGGCCAGTGGCTTGGGATG \\
\hline \multirow[t]{7}{*}{ GABAergic } & Ionotropic & $\begin{array}{c}\gamma \text {-amino } \\
\text { butyric acid }\end{array}$ & GABRA3 & GCAGAACTGCACTCTGGAAA & ACTCCGGTAACAGCCTTGTC \\
\hline & Ionotropic & ATP & $P 2 X R 1$ & TTGTGGAGAACGGGACCAA & GTCAAAGCGAATCCCAAACAC \\
\hline & & & $P 2 X R 3$ & GCATCCCCAAATACTCCTTCAC & GGACACGCTGCTITTCTCAGA \\
\hline & & & $P 2 X R 4$ & GCCGCCTCGATACACGGGAC & TGCTCGTTGCCAGCCAGGTC \\
\hline & & & P2XR5 & СCTTCCTGCCAGCTGTTTG & TGCCATCTCCCCCACTTTAA \\
\hline & & & P2XR6 & CCAAACAACACCACCGAGATC & TGGGACCAAGAGGAGAATTCC \\
\hline & & & $P 2 X R 7$ & ACTGCAACCTAGACCGTTGGTT & TCAAGGCGACGGAAACTGTAT \\
\hline & Metabotropic & & P2YR1 & GGATGCCATGTGTAAACTGC & GTACACCACACCGCTGTACC \\
\hline & & & P2YR2 & CACCCGCACCCTCTACTACT & CCTTGTAGGCCATGTTGATG \\
\hline & & & P2YR6 & GCCGGCGACCACATGA & CCTGCCTCTGCCATTTGG \\
\hline & & & P2YR12 & AATGCAAGCCGTCGACAAC & CTCTGGTGCACAGACTGGTGT \\
\hline & & & P2YR13 & GTGCCACGAGCTCCAACAC & TGAGGCCATGGAAGAAAACG \\
\hline & & & P2YR14 & TCTTCATTGCAGGAATCCTACTCA & AGAGCTGGGCACGTAAAAGAAT \\
\hline \multirow[b]{2}{*}{ Kininergic } & & & $B D K R B 1$ & TCAGCAGGACAAGGTGCGGG & GGCCCAGCAGACCAGGAAGG \\
\hline & Metak & Brady & BDKRB2 & TGGTGTTCCGGACCATGAAG & AAGCCCACGACATTCAGGAAG \\
\hline
\end{tabular}

Publisher's Note Springer Nature remains neutral with regard to jurisdictional claims in published maps and institutional affiliations. 